\section{Public funding of uterine transplantation}

\author{
Jacques Balayla
}

The recent success of uterine transplantation (UTx) leading to a viable birth has provided the first evidence for the treatment and cure of absolute uterine factor infertility. ${ }^{1}$ Indeed, while its clinical and scientific merits have been recently established, ${ }^{1}$ a number of social, economic and ethical concerns remain. ${ }^{2-4}$ In particular, the question whether uterine transplants should be publicly funded remains a source of debate and controversy. ${ }^{5}$

In an insightful essay by Nicola Williams and Stephen Wilkinson, the authors address the question from the opposing premise: are there any compelling reasons for the state not to fund UTx $?^{5}$ To achieve this goal, the authors counter three arguments commonly raised against the public funding of UTx. The first argument suggests that UTx funding would increase the carbon footprint, which is inconsistent with governments' obligations to prevent climate change. The second claims that UTx does not treat a disorder and is therefore not medically necessary. Finally, the third asserts that funding for UTx should be denied because of the availability of alternatives such as adoption and surrogacy.

Before undertaking their analysis, two key premises are raised by the authors. First, their study is limited to countries with socialised healthcare systems paid through taxation and may not apply to countries where healthcare is privately funded. This argument is a key to the understanding of the ethical implications of UTx funding at the world's stage. Second, and perhaps more importantly, an emphasis is placed on the notion that before determining the source of funding for UTx, its clinical safety and efficacy must be established. ${ }^{5}$ Indeed, 'The Montreal Criteria for the Ethical Feasibility of Uterine Transplantation' are in agreement with such imposition. ${ }^{3}$ In this regard, the authors' work may be somewhat premature. As it has been done in its experimental stage, where it still remains, UTx programmes are better funded by grants from private donations, and are better undertaken outside of

Correspondence to Dr Jacques Balayla, Obstetrics and Gynecology, University of Montreal, Montreal, Quebec, Canada H4W1S8; jacques.balayla@gmail.com working hours so as not to interfere with resource allocation for patients covered by the system. ${ }^{6}$

To begin, the environmental argument is problematic. It is well known that patients with disabilities are already disproportionately stigmatised based largely on their condition. ${ }^{8}$ Adding undue burden and discrimination about their potential carbon footprint as they try to integrate into Society, be it by having children or benefiting from Motability, would arguably be immoral and in violation of Equity. One could argue that providing life-saving treatment to an individual who would otherwise perish is also increasing environmental emissions as that individual continues to live. Yet, it would be highly unethical to defund care based on that premise. In the case of UTx, the incidence of uterine factor infertility is estimated at $1 / 5000$, of which only a minority of individuals in their reproductive years would theoretically seek the transplant. In the grand scheme of things, where industrial and military emissions are in part publicly funded, any intervention on the UTx population is unlikely to have a major relative impact on our carbon footprint.

With regard to the argument against the true nature of infertility as a disease, there should be no question that by any standard, infertility is a disease with a biological cause. The reasons are straightforward. First, one can induce infertility by disrupting the biological mechanisms of the body, and second, one is not labelled as 'infertile' unless a work-up has been undertaken, and a diagnosis made. From this argument, it follows that a number of individuals who have failed to conceive in 12 months, and who are thus technically 'infertile' as per the WHO definition, may not label themselves as such until someone with the expertise does. These individuals do not seek treatment for a variety of reasons, some of which include no desire to procreate or no access to care. However, in neither case is the nature of infertility as a disease put into question.

From the argument that social context and individual situations make it such that infertility is only 'harmful to people with certain desires' it does not follow that infertility is therefore not a disease. ${ }^{5}$ The same principle is beautifully paralleled in psychiatric diagnoses. In the Diagnostic and Statistical Manual of Mental Disorders (DSM) system, making a diagnosis depends on a conjoint assessment of symptoms and functioning. ${ }^{9}$ In other words, an individual meeting criteria for depression, who is nevertheless functional or not bothered by such symptoms, is technically not considered as being depressed. Indeed, akin to infertility, though the subjective experience of disease dictates treatment, it never questions the true nature of the condition.

In their text, the authors cite Pemberton, who considers that infertility brings about a 'grief based on a sense of failure because of an "abnormality" that is culturally determined'.5 In effect, Pemberton argues that infertility leads to agony and sorrow because an infecund couple fails to meet cultural rather than biological standards. Such argument is inaccurate and misleading. The biological imperative, defined as the innate drive of living organisms to perpetuate their existence, is largely evolutionary in nature and predates modern cultural norms. The biological imperative opposes a primarily social drive for reproduction of the species, and is therefore inconsistent with Pemberton's argument. Indeed, while infertility does bring about social consequences, they are rooted in perceived biological failures.

That infertility is perceived as a purely social issue is frequently used to parallel cosmetic surgery and fertility treatments, and used as the pinnacle to oppose public funding for both. Funding of elective aesthetic procedures that enhance body image for primarily social purposes should not be compared with the funding of fertility treatments. Indeed, while the 'desired' or 'ideal' body type has largely changed throughout history to meet cultural and social standards, the same cannot be said about procreation, for it would violate the very basic principle of evolutionary biology.

Finally, one could argue that the whole premise of the argument that there exist alternatives to UTx is flawed. The question that must be first addressed is thus: what is the primary goal of UTx? Is it to allow an infertile person to become a parent? No clinician would argue that UTx is potentially superior to adoption or surrogacy in terms of efficacy, safety or expediency in this respect. ${ }^{3}$ However, the proper question is whether UTx is superior in allowing a woman the opportunity to carry a pregnancy. In this respect, UTx has the full potential for superiority, given 
that surrogacy and adoption are not real alternatives. ${ }^{3}$ The authors argue that experiencing gestation may be relatively unimportant compared with being a social parent from birth to certain individuals. Such nuanced interpretation may be problematic for women who see the process of conception to delivery and raising of the family as one sacred continuum. In this regard, UTx may be beneficial over the two alternatives. Lastly, with regard to cost, it remains uncertain that commercial surrogacy arrangements, where available, are less costly than UTx. Thus, is it not entirely clear that surrogacy and adoption are always viable, let alone 'sufficiently good' alternatives to UTx.

All in all, the authors bring forth a convincing case that the aforementioned arguments against state-funded UTx are weak. The aspects and discussion hereby conveyed are fundamental to the advancement of the UTx cause and crucial before its widespread application.
Contributors JB is the sole author and contributor to this piece.

Competing interests None declared.

Patient consent No.

Provenance and peer review Not commissioned; internally peer reviewed.

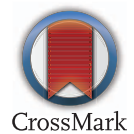

To cite Balayla J. J Med Ethics 2016;42:568-569.

Received 30 November 2015

Revised 29 December 2015

Accepted 15 January 2016

Published Online First 8 February 2016

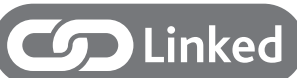

- http://dx.doi.org/10.1136/medethics-2015-102999

J Med Ethics 2016;42:568-569.

doi:10.1136/medethics-2015-103232

\section{REFERENCES}

1 Brännström $\mathrm{M}$, Johannesson $\mathrm{L}$, Bokström $\mathrm{H}$, et al. Livebirth after uterus transplantation. Lancet 2015;385:607-16.

2 Lefkowitz A, Edwards M, Balayla J. Ethical considerations in the era of the uterine transplant: an update of the Montreal Criteria for the ethical feasibility of uterine transplantation. Fertil Steril 2013;100:924-6.

3 Lefkowitz A, Edwards M, Balayla J. The Montreal Criteria for the ethical feasibility of uterine transplantation. Transp/ Int 2012;25:439-47.

4 Caplan AL. Ensuring the future of uterine transplantation. Fertil Steril 2013;99:682-3.

5 Williams N, Wilkinson S. Should uterus transplants be publicly funded? Journal of Medical Ethics, 2015.

6 Brännström $\mathrm{M}$, Johannesson $\mathrm{L}$, Dahm-Kähler $\mathrm{P}$, et al. First clinical uterus transplantation trial: a six-month report. Fertil Steril 2014;101:1228-36.

7 Johannesson L, Kvarnström N, Mölne J, et al. Uterus transplantation trial: 1-year outcome. Fertil Steril 2015;103:199-204.

8 Slade P, O'Neill C, Simpson AJ, et al. The relationship between perceived stigma, disclosure patterns, support and distress in new attendees at an infertility clinic. Hum Reprod 2007;22:2309-17.

9 Ustün B, Kennedy C. What is "functional impairment"? Disentangling disability from clinical significance. World Psychiatry 2009;8:82-5. 